\title{
On a Generalized Integro-Differential Equation of Barbashin Type
}

\author{
Chen Chur-jen
}

\begin{abstract}
We study a class of integro-differential equations containing multiplication operators, partial integral operators, and ordinary integral operators. Building on the usual identification of real functions of several variables and abstract functions, such integro-differential equations may be reformulated as ordinary differential equations in suitable Banach spaces. We give a representation theorem for the corresponding Cauchy operator and study the (unique) solvability of a general boundary value problem.

Keywords: Integro-differential equations of Barbashin type, differential equations in Banach spaces, partial integral operators, Cauchy operators, boundary value problems, spectral radius, ideal spaces
\end{abstract}

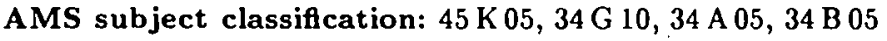

\section{Generalized equations of Barbashin type}

Let $J \subset \mathbb{R}$ be some compact interval and let

$$
\begin{aligned}
c & :[a, b] \times J \times[a, b] \rightarrow \mathbb{R} \\
l & :[a, b] \times J \times[a, b] \times[a, b] \rightarrow \mathbb{R} \\
m & :[a, b] \times J \times[a, b] \times[a, b] \rightarrow \mathbb{R} \\
n & :[a, b] \times J \times[a, b] \times[a, b] \times[a, b] \rightarrow \mathbb{R} \\
f: & {[a, b] \times J \times[a, b] \rightarrow \mathbb{R} }
\end{aligned}
$$

be measurable functions. In what follows, we consider the integro-differential equation

$$
\begin{aligned}
\frac{\partial u(t, \tau, s)}{\partial \tau}= & c(t, \tau, s) u(t, \tau, s) \\
& +\int_{a}^{b} l(t, \tau, s, \sigma) u(t, \tau, \sigma) d \sigma+\int_{a}^{b} m(t, \tau, s, r) u(r, \tau, s) d r
\end{aligned}
$$

Chen Chur-jen: Tunghai University, Dep. Math., Taichung, Taiwan. Current address: Universität Würzburg, Math. Inst., Am Hubland, D - 97074 Würzburg.

This paper was written while the author was visiting the University of Würzburg as a DAAD fellow. Financial support by the DAAD Bonn (Kz. A/95/08858) is gratefully acknowledged. 


$$
+\int_{a}^{b} \int_{a}^{b} n(t, \tau, s, \sigma, r) u(r, \tau, \sigma) d \sigma d r+f(t, \tau, s) .
$$

Equations of this type are usually called generalized integro-differential equations of Barbashin type. They arise in the mathematical modelling of certain transport and radiation phenomena $[7,9]$ as well as in acoustic scattering theory [3].

In order to study equation (1) in a general functional-analytic setting, it is a useful device to write (1) as an operator differential equation in some Banach space. To this end, let $X$ be some ideal space over $[a, b] \times[a, b]$, i.e. a Banach space of measurable real functions on $[a, b] \times[a, b]$ with monotone norm, i.e. if $y \in X$ and $x$ is measurable with $|x| \leq|y|$ a.e., then also $x \in X$ and $\|x\| \leq\|y\|$ (see, e.g., [11]). We denote by $C(J, X)$ and $C^{1}(J, X)$ the spaces of all continuous and all continuously differentiable abstract functions $u: J \rightarrow X$, respectively. Moreover, we write $C_{\tau}(X)$ for the space of all measurable functions $u:[a, b] \times J \times[a, b] \rightarrow \mathbb{R}$ such that the map $\tau \mapsto u(\cdot, \tau, \cdot)$ belongs to $C(J, X)$, and $C_{\tau}^{1}(X)$ for the space of all functions $u:[a, b] \times J \times[a, b] \rightarrow \mathbb{R}$ such that the map $u(t, \cdot, s)$ is absolutely continuous on $J$ and $\frac{\partial u}{\partial r} \in C_{r}(X)$.

We usually identify functions of $C(J, X)$ and $C_{T}(X)$ by putting

$$
u(\tau)(t, s)=u(t, \tau, s)
$$

In this sense we can prove (see also $[2,5]$ ) the following

Lemma 1. With the natural identification (2), we have $C(J, X) \cong C_{\tau}(X)$ and $C^{1}(J, X) \cong C_{\tau}^{1}(X)$. Furthermore, for $u \in C_{r}^{1}(X)$ we have

$$
\frac{d u(\tau)}{d \tau}(t, s)=\frac{\partial u(t, \tau, s)}{\partial \tau}
$$

and each $u \in C_{r}(X)$ satisfies

$$
\left(\int_{J} u(\tau) d \tau\right)(t, s)=\int_{J} u(t, \tau, s) d \tau
$$

for almost all $(t, s) \in[a, b] \times[a, b]$.

Using the identification (2), we may write equation (1) more concisely as operator differential equation

$$
\frac{d u(\tau)}{d \tau}=A(\tau) u(\tau)+f(\tau) \quad(\tau \in J)
$$

where

$$
A(\tau)=C(\tau)+K(\tau), \quad K(\tau)=L(\tau)+M(\tau)+N(\tau)
$$

with

$$
\begin{aligned}
& C(\tau) u(t, s)=c(t, \tau, s) u(t, s) \\
& L(\tau) u(t, s)=\int_{a}^{b} l(t, \tau, s, \sigma) u(t, \sigma) d \sigma
\end{aligned}
$$




$$
\begin{aligned}
& M(\tau) u(t, s)=\int_{a}^{b} m(t, \tau, s, r) u(r, s) d r \\
& N(\tau) u(t, s)=\int_{a}^{b} \int_{a}^{b} n(t, \tau, s, \sigma, r) u(r, \sigma) d \sigma d r .
\end{aligned}
$$

Any solution $u \in C^{1}(J, X)$ of equation (3) will then be called a solution of the integrodifferential equation (1) in the ideal space $X$. In applications, the usual choice for $X$ is either the Lebesgue space $L_{p}=L_{p}([a, b] \times[a, b])$ or, more generally, the Orlicz space $L_{M}=L_{M}([a, b] \times[a, b])$ generated by some Young function $M$ (see, e.g., [8]).

\section{A representation theorem for the Cauchy operator}

The Cauchy operator (or resolvent operator) plays a crucial role in the classical theory of differential equations in Banach spaces (see, e.g., [4]). However, there is a problem when applying the classical theory to equation (3): the operators (6) and (7) (which may be called partial integral operators, since integration is carried out only with respect to some of the variables involved) are usually not compact. Nevertheless, the central representation theorem for the Cauchy operator carries over from integral to partial integral operators, as we shall show now.

We define $\mathcal{L}^{r}(X)$ as the space of all regular partial integral operators (see [10]) in $X$. This is a Banach space with the norm

$$
\|A\|_{\mathcal{L}(X)}=\||A|\|_{\mathcal{C}(X)},
$$

where $|A|$ denotes the module of $A$ (see again [10]). It turns out that, if (4) is locally (Bochner) integrable as a function from some interval into $\mathcal{L}^{r}(X)$, the Cauchy operator for equation (3) is itself a regular partial integral operator:

Theorem 1. Suppose that the operator function (4) is locally integrable in $\mathcal{L}^{r}(X)$. Then the Cauchy operator $U\left(\tau, \tau_{0}\right)$ for the differential equation (3) admits a representation

$$
U\left(\tau, \tau_{0}\right) u(t, s)=e\left(t, \tau, \tau_{0}, s\right) u(t, s)+H\left(\tau, \tau_{0}\right) u(t, s)
$$

where

$$
e\left(t, \tau, \tau_{0}, s\right)=\exp \left\{\int_{r_{0}}^{r} c(t, \xi, s) d \xi\right\}
$$

and $H$ is a partial integral operator defined by

$$
\begin{aligned}
H\left(\tau, \tau_{0}\right) u(t, s)= & \int_{a}^{b} \hat{l}\left(t, \tau, \tau_{0}, s, \sigma\right) u(t, \sigma) d \sigma+\int_{a}^{b} \hat{m}\left(t, \tau, \tau_{0}, s, r\right) u(r, s) d r \\
& +\int_{a}^{b} \int_{a}^{b} \hat{n}\left(t, \tau, \tau_{0}, s, \sigma, r\right) u(r, \sigma) d \sigma d r
\end{aligned}
$$


with measurable functions

$$
\begin{gathered}
\hat{l}:[a, b] \times J \times J \times[a, b] \times[a, b] \rightarrow \mathbb{R} \\
\hat{m}:[a, b] \times J \times J \times[a, b] \times[a, b] \rightarrow \mathbb{R} \\
\hat{n}:[a, b] \times J \times J \times[a, b] \times[a, b] \times[a, b] \rightarrow \mathbb{R} .
\end{gathered}
$$

Moreover, we have the inclusion $U\left(\tau, \tau_{0}\right) \in \mathcal{L}^{r}(X)$ and the implications

$$
\begin{aligned}
& l=0 \quad \Longrightarrow \quad \hat{l}=0 \\
& m=0 \quad \Longrightarrow \quad \hat{m}=0 \\
& n=l=0 \quad \Longrightarrow \quad \hat{n}=\hat{l}=0 \\
& n=m=0 \quad \Longrightarrow \quad \hat{n}=\hat{m}=0 \text {. }
\end{aligned}
$$

Finally, if $A$ is of Volterra type, i.e.

$$
\begin{aligned}
& l(t, \tau, s, \sigma)=0 \quad(\sigma>s), \quad m(t, \tau, s, r)=0 \quad(r>t) \\
& n(t, \tau, s, \sigma, r)=0 \quad(\sigma>s \quad \text { or } r>t),
\end{aligned}
$$

then $H$ is also of Volterra type.

Proof. The Cauchy operator $U\left(\tau, \tau_{0}\right)$ is given by the series

$$
U\left(\tau, \tau_{0}\right)=I+\sum_{n=1}^{\infty} \int_{\tau_{0}}^{\tau} \int_{\tau_{0}}^{\tau_{1}} \int_{\tau_{0}}^{\tau_{2}} \cdots \int_{\tau_{0}}^{\tau_{n-1}} A\left(\tau_{1}\right) A\left(\tau_{2}\right) \cdots A\left(\tau_{n}\right) d \tau_{n} \cdots d \tau_{1}
$$

which converges in the norm of the space $\mathcal{L}^{r}(X)$. By [6: Theorems 7 and 8], every operator $A\left(\tau_{1}\right) A\left(\tau_{2}\right) \cdots A\left(\tau_{n}\right) \quad(n=1,2, \ldots)$ belongs to the space $\mathcal{R}_{c}(X)+\mathcal{R}_{l}(X)+$ $\mathcal{R}_{m}(X)+\mathcal{R}_{n}(X)$, i.e. admits a representation

$$
\begin{aligned}
A\left(\tau_{1}\right) \cdots A\left(\tau_{n}\right) u(t, s)= & c\left(t, \tau_{1}, s\right) \cdots c\left(t, \tau_{n}, s\right) u(t, s) \\
& +\int_{a}^{b} l_{n}\left(t, \tau_{1}, \ldots, \tau_{n}, s, \sigma\right) u(t, \sigma) d \sigma \\
& +\int_{a}^{b} m_{n}\left(t, \tau_{1}, \ldots, \tau_{n}, s, r\right) u(r, s) d r \\
& +\int_{a}^{b} \int_{a}^{b} n_{n}\left(t, \tau_{1}, \ldots, \tau_{n}, s, \sigma, r\right) u(r, \sigma) d \sigma d r
\end{aligned}
$$

where the kernel functions are all measurable. Moreover,

$$
\begin{aligned}
\left\|l_{n}\left(\cdot, \tau_{1}, \ldots, \tau_{n}, \cdot, \cdot\right)\right\|_{\mathcal{R}_{I}(X)} & \leq\left\|A\left(\tau_{1}\right)\right\|_{\mathcal{L}^{r}(X)} \cdots\left\|A\left(\tau_{n}\right)\right\|_{\mathcal{L}^{r}(X)} \\
\left\|m_{n}\left(\cdot, \tau_{1}, \ldots, \tau_{n}, \cdot, \cdot\right)\right\|_{\mathcal{R}_{m}(X)} & \leq\left\|A\left(\tau_{1}\right)\right\|_{\mathcal{L}^{r}(X)} \cdots\left\|A\left(\tau_{n}\right)\right\|_{\mathcal{L}^{r}(X)}, \\
\left\|n_{n}\left(\cdot, \tau_{1}, \ldots, \tau_{n}, \cdot, \cdot, \cdot\right)\right\|_{\mathcal{R}_{n}(X)} & \leq\left\|A\left(\tau_{1}\right)\right\|_{\mathcal{L}^{r}(X)} \cdots\left\|A\left(\tau_{n}\right)\right\|_{\mathcal{L}^{r}(X)} .
\end{aligned}
$$


In fact, it is obvious by [6: Theorem 2] and (9) that the left-hand sides of the previous inequalities are all bounded by

$$
\begin{aligned}
\left\|\left|A\left(\tau_{1}\right) \cdots A\left(\tau_{n}\right)\right|\right\|_{\mathcal{L}(X)} & \leq\left\|\left|A\left(\tau_{1}\right)\right| \cdots\left|A\left(\tau_{n}\right)\right|\right\|_{\mathcal{L}(X)} \\
& \leq\left\|\left|A\left(\tau_{1}\right)\right|\right\|_{\mathcal{L}(X)} \cdots\left\|\left|A\left(\tau_{n}\right)\right|\right\|_{\mathcal{L}(X)} \\
& =\left\|A\left(\tau_{1}\right)\right\|_{\mathcal{L}^{r}(X)} \cdots\left\|A\left(\tau_{n}\right)\right\|_{\mathcal{C}^{r}(X)} .
\end{aligned}
$$

Integrating (9) over $\tau_{1}, \ldots, \tau_{n}$ yields

$$
\begin{aligned}
\left(\int_{\tau_{0}} \int_{\tau_{0}}^{\tau_{1}} \ldots\right. & \left.\int_{\tau_{0}}^{\tau_{n}-1} A\left(\tau_{1}\right) \ldots A\left(\tau_{n}\right) d \tau_{n} \cdots d \tau_{1}\right) u(t, s) \\
= & \frac{1}{n !}\left(\log e\left(t, \tau, \tau_{0}, s\right)\right)^{n} u(t, s) \\
& +\int_{a}^{b}\left(\int_{\tau_{0}}^{r} \ldots \int_{\tau_{0}}^{\tau_{n}-1} l_{n}\left(t, \tau_{1}, \ldots, \tau_{n}, s, \sigma\right) d \tau_{n} \cdots d \tau_{1}\right) u(t, \sigma) d \sigma \\
& +\int_{a}^{b}\left(\int_{\tau_{0}}^{\tau} \ldots \int_{\tau_{0}}^{\tau_{n}-1} m_{n}\left(t, \tau_{1}, \ldots, \tau_{n}, s, r\right) d \tau_{n} \cdots d \tau_{1}\right) u(r, s) d r \\
& +\int_{a}^{b} \int_{a}^{\tau_{n-1}}\left(\int_{\tau_{0}}^{\tau} \ldots \int_{\tau_{0}}^{\tau_{n}} n_{n}\left(t, \tau_{1}, \ldots, \tau_{n}, s, \sigma, r\right) d \tau_{n} \cdots d \tau_{1}\right) u(r, \sigma) d \sigma d r
\end{aligned}
$$

where we made use of Fubini's theorem and Lemma 1. Since the spaces $\mathcal{R}_{l}(X), \mathcal{R}_{m}(X)$, and $\mathcal{R}_{n}(X)$ are closed, we may interchange summation and integration. Thus the assertion follows with

$$
\begin{aligned}
\hat{l}\left(t, \tau, \tau_{0}, s, \sigma\right) & =\sum_{n=1}^{\infty} \int_{\tau_{0}}^{\tau} \ldots \int_{\tau_{0}}^{\tau_{n}-1} l_{n}\left(t, \tau_{1}, \ldots, \tau_{n}, s, \sigma\right) d \tau_{n} \cdots d \tau_{1} \\
\hat{m}\left(t, \tau, \tau_{0}, s, r\right) & =\sum_{n=1}^{\infty} \int_{\tau_{0}}^{\tau} \cdots \int_{\tau_{0}-1}^{\tau_{n}} m_{n}\left(t, \tau_{1}, \ldots, \tau_{n}, s, r\right) d \tau_{n} \cdots d \tau_{1} \\
\hat{n}\left(t, \tau, \tau_{0}, s, \sigma, r\right) & =\sum_{n=1}^{\infty} \int_{\tau_{0}}^{\tau} \cdots \int_{\tau_{0}}^{\tau_{n}-1} n_{n}\left(t, \tau_{1}, \ldots, \tau_{n}, s, \sigma, r\right) d \tau_{n} \cdots d \tau_{1} .
\end{aligned}
$$

More precisely, we have by [6] (eliminating some typing errors) that $l_{1}=l, m_{1}=m$, 
$n_{1}=n$

$$
\begin{aligned}
& l_{n+1}\left(t, \tau_{1}, \ldots, \tau_{n+1}, s, \sigma\right) \\
& =c\left(t, \tau_{1}, s\right) \cdots c\left(t, \tau_{n}, s\right) l\left(t, \tau_{n+1}, s, \sigma\right) \\
& +l_{n}\left(t, \tau_{1}, \ldots, \tau_{n}, s, \sigma\right) c\left(t, \tau_{n+1}, \sigma\right) \\
& +\int_{a}^{b} l_{n}\left(t, \tau_{1}, \ldots, \tau_{n}, s, \xi\right) l\left(t, \tau_{n+1}, \xi, \sigma\right) d \xi \\
& m_{n+1}\left(t, \tau_{1}, \ldots, \tau_{n+1}, s, r\right) \\
& =c\left(t, \tau_{1}, s\right) \cdots c\left(t, \tau_{n}, s\right) m\left(t, \tau_{n+1}, s, r\right) \\
& +m_{n}\left(t, \tau_{1}, \ldots, \tau_{n}, s, r\right) c\left(r, \tau_{n+1}, s\right) \\
& +\int_{a}^{b} m_{n}\left(t, \tau_{1}, \ldots, \tau_{n}, s, \eta\right) m\left(\eta, \tau_{n+1}, s, r\right) d \eta \\
& n_{n+1}\left(t, \tau_{1}, \ldots, \tau_{n+1}, s, \sigma, r\right) \\
& =c\left(t, \tau_{1}, s\right) \cdots c\left(t, \tau_{n}, s\right) n\left(t, \tau_{n+1}, s, \sigma, r\right) \\
& +n_{n}\left(t, \tau_{1}, \ldots, \tau_{n}, s, \sigma, r\right) c\left(r, \tau_{n+1}, \sigma\right) \\
& +l_{n}\left(t, \tau_{1}, \ldots, \tau_{n}, s, \sigma\right) m\left(t, \tau_{n+1}, \sigma, r\right) \\
& +m_{n}\left(t, \tau_{1}, \ldots, \tau_{n}, s, r\right) l\left(r, \tau_{n+1}, s, \sigma\right) \\
& +\int_{a}^{b} l_{n}\left(t, \tau_{1}, \ldots, \tau_{n}, s, \xi\right) n\left(t, \tau_{n+1}, \xi, \sigma, r\right) d \xi \\
& +\int_{a}^{b} m_{n}\left(t, \tau_{1}, \ldots, \tau_{n}, s, \eta\right) n\left(\eta, \tau_{n+1}, s, \sigma, r\right) d \eta \\
& +\int_{a}^{b} n_{n}\left(t, \tau_{1}, \ldots, \tau_{n}, s, \xi, r\right) l\left(r, \tau_{n+1}, \xi, \sigma\right) d \xi \\
& +\int_{a}^{b} n_{n}\left(t, \tau_{1}, \ldots, \tau_{n}, s, \sigma, \eta\right) m\left(\eta, \tau_{n+1}, \sigma, r\right) d \eta \\
& +\int_{a}^{b} \int_{a}^{b} n_{n}\left(t, \tau_{1}, \ldots, \tau_{n}, s, \xi, \eta\right) n\left(\eta, \tau_{n+1}, \xi, \sigma, r\right) d \eta d \xi .
\end{aligned}
$$

By these formulas, the additional statements are obvious

As can be seen by the formula for $n_{n+1}$, the implication " $n=0 \Longrightarrow \hat{n}=0$ " is in 
general not true, if $l$ and $m$ do not vanish.

Of course, the formulas arising in the proof of Theorem 1 are very clumsy, and they may hardly be used for calculating the Cauchy operator $U\left(\tau, \tau_{0}\right)$. Under additional assumptions, however, the calculations simplify. For example, in case $m=n=0$ (or, analogously, in case $l=n=0$ ) equation (3) becomes a usual Barbashin equation with respect to $(\tau, s)$ involving a parameter $t$. In this case we consider the parameter dependent operator function

$$
A_{t}(\tau) v(s)=c(t, \tau, s) v(s)+\int_{a}^{b} l(t, \tau, s, \sigma) v(\sigma) d \sigma
$$

and assume that we are able to calculate the Cauchy operator for the Barbashin equation

$$
\frac{d v(\tau)}{d \tau}=A_{t}(\tau) v(\tau) \quad(\tau \in J)
$$

in some Banach space $Y$ over $[a, b]$ which is obtained from $X$ by "freezing" the argument $t$. In order to make this more precise, let us say that an ideal space $X$ over $[a, b] \times[a, b]$ is projectable to an ideal space $Y$ over $[a, b]$, if for any $u \in X$ we have $u(t, \cdot) \in Y$ for almost all $t \in[a, b]$.

For example, $X=L_{p}([a, b] \times[a, b])$ is always projectable to $Y=L_{p}([a, b])$. More generally, for ideal spaces $X$ with mixed norm (see, e.g., [6]), it is trivial to determine a space $Y$ to which $X$ is projectable.

Theorem 2. Let $m=n=0$. Suppose that $X$ is projectable to $Y$, and the operator function (4) is locally integrable in $\mathcal{L}(X)$. Assume that, for almost all $t \in[a, b]$, the operator function $(10)$ is locally integrable in $\mathcal{L}(Y)$ with Cauchy operator $U_{t}\left(\tau, \tau_{0}\right)$. Then the Cauchy operator of equation (3) is given by

$$
U\left(\tau, \tau_{0}\right) u(t, s)=U_{t}\left(\tau, \tau_{0}\right) u(t, \cdot)(s)
$$

where equality holds for almost all $t \in[a, b]$, provided one chooses a proper representation of the right-hand side.

Proof. Again, the Cauchy operators $U\left(\tau, \tau_{0}\right)$ and $U_{\imath}\left(\tau, \tau_{0}\right)$ can be calculated by

$$
\begin{aligned}
& U\left(\tau, \tau_{0}\right) u=u+\sum_{n=1}^{\infty} \int_{\tau_{0}}^{\tau} \int_{\tau_{0}}^{\tau_{1}} \int_{\tau_{0}}^{\tau_{2}} \ldots \int_{\tau_{0}}^{\tau_{n-1}} A\left(\tau_{1}\right) A\left(\tau_{2}\right) \cdots A\left(\tau_{n}\right) u d \tau_{n} \ldots d \tau_{1} \\
& U_{t}\left(\tau, \tau_{0}\right) v=v+\sum_{n=1}^{\infty} \int_{\tau_{0}}^{\tau} \int_{\tau_{0}}^{\tau_{1}} \int_{\tau_{0}}^{\tau_{2}} \cdots \int_{\tau_{0}}^{\tau_{n-1}} A_{t}\left(\tau_{1}\right) A_{t}\left(\tau_{2}\right) \cdots A_{t}\left(\tau_{n}\right) v d \tau_{n} \cdots d \tau_{1} .
\end{aligned}
$$

Now observe that for any $u \in X$ we have $A(\tau) u(t, s)=A_{t}(\tau) u(t, \cdot)(s)$ for almost all $(t, s) \in[a, b] \times[a, b]$. Consequently, by induction the equality

$$
A\left(\tau_{1}\right) A\left(\tau_{2}\right) \cdots A\left(\tau_{n}\right) u(t, s)=A_{t}\left(\tau_{1}\right) A_{t}\left(\tau_{2}\right) \cdots A_{t}\left(\tau_{n}\right) u(t, \cdot)(s)
$$

holds for almost all $(t, s) \in[a, b] \times[a, b]$. By Lemma 1 (and an analogous result for $Y)$, the partial sums of (12) and (13) for $U\left(\tau, \tau_{0}\right) u(t, \cdot)$ and $U_{t}\left(\tau, \tau_{0}\right) u(t, \cdot)$ coincide, for almost all $t$, a.e. on $[a, b]$. It remains to observe that, by the following lemma, the same is true for their limits 
Lemma 2. Let $\Omega$ be a finite measure space and $X$ an ideal space over $\Omega \times \Omega$ which is projectable to some ideal space $Y$ over $\Omega$. If $\left\|u_{n}-u\right\|_{X} \rightarrow 0$, and $\left\|u_{n}(t, \cdot)-v(t, \cdot)\right\|_{Y} \rightarrow 0$ for almost all $t \in \Omega$, then for almost all $t$ we have $u(t, \cdot)=v(t, \cdot)$ a.e. on $\Omega$.

Proof. By the assumption on $\Omega$, we have $u_{n} \rightarrow u$ in measure, hence for a properly chosen subsequence $u_{n_{k}} \rightarrow u$ a.e. on $\Omega$. In other words, for almost all $t$ we have $u_{n_{k}}(t, \cdot) \rightarrow u(t, \cdot)$ a.e. on $\Omega$, and hence also in measure. On the other hand, we have $u_{n}(t, \cdot) \rightarrow v(t, \cdot)$ in measure for almost all $t \in \Omega$

We illustrate the results of this section by means of an elementary example.

Example 1. Let $[a, b]=[0,1], X=L_{2}, c(t, \tau, s)=m(t, \tau, s, r)=n(t, \tau, s, \sigma, r)=$ $f(t, \tau, s) \equiv 0$, and $l(t, \tau, s, \sigma)=\tau$, i.e. we consider the differential equation (3) with $f(\tau) \equiv 0$ and

$$
A(\tau) u(t, s)=\int_{0}^{1} \tau u(t, \sigma) d \sigma .
$$

It is obviously that $A(\tau)$ is locally integrable in $\mathcal{L}^{r}(X)$. According to the proof of Theorem 1, we get

$$
\begin{aligned}
m_{n}\left(t, \tau_{1}, \ldots, \tau_{n}, s, r\right) & =n_{n}\left(t, \tau_{1}, \ldots, \tau_{n}, s, \sigma, r\right) \equiv 0 \\
l_{n}\left(t, \tau_{1}, \ldots, \tau_{n}, s, \sigma\right) & =\tau_{1} \tau_{2} \cdots \tau_{n} .
\end{aligned}
$$

This implies that

$$
\begin{aligned}
\hat{m}\left(t, \tau, \tau_{0}, s, r\right) & =\hat{n}\left(t, \tau, \tau_{0}, s, \sigma, r\right) \equiv 0 \\
\hat{l}\left(t, \tau, \tau_{0}, s, \sigma\right) & =e^{\tau-\tau_{0}}-1 .
\end{aligned}
$$

The Cauchy operator $U\left(\tau, \tau_{0}\right)$ is therefore given in this case by

$$
U\left(\tau, \tau_{0}\right) u(t, s)=u(t, s)+H\left(\tau, \tau_{0}\right) u(t, s)=u(t, s)+\int_{0}^{1}\left(e^{r-\tau_{0}}-1\right) u(t, \sigma) d \sigma
$$

On the other hand, the corresponding parameter dependent operator function (10) is here

$$
A_{t}(\tau) v(s)=\int_{0}^{1} \tau v(\sigma) d \sigma
$$

By Theorem 2, the Cauchy operator for the differential equation

$$
\frac{d v(\tau)}{d \tau}=A_{t}(\tau) v(\tau) \quad(\tau \in J)
$$

has the form

$$
U_{t}\left(\tau, \tau_{0}\right) v(s)=v(s)+\int_{0}^{1}\left(e^{\tau-\tau_{0}}-1\right) v(\sigma) d \sigma
$$

and the equality $U\left(\tau, \tau_{0}\right) u(t, s)=U_{t}\left(\tau, \tau_{0}\right) u(t, \cdot)(s)$ for almost all $t \in[0,1]$ is obvious. 


\section{Generalized boundary value problems}

We consider now the equation (1) for $(t, \tau, s) \in[-1,1] \times\left[\tau_{1}, \tau_{2}\right] \times[-1,1]$, subject to the boundary conditions

$$
\begin{array}{ll}
u\left(t, \tau_{1}, s\right)=\varphi(t, s) & ((t, s) \in Q) \\
u\left(t, \tau_{2}, s\right)=\psi(t, s) & \left((t, s) \in Q^{c}=([-1,1] \times[-1,1]) \backslash Q\right)
\end{array}
$$

where $Q$ is some measurable subset of $[-1,1] \times[-1,1]$. As before, let $X$ be an ideal space over $[-1,1] \times[-1,1]$. A solution of this boundary value problem is, by definition, a function $u \in C_{r}^{1}(X)$ which satisfies (1), (14) and (15) almost everywhere. In the special case

$$
c(t, \tau, s)=c(s), \quad l(t, \tau, s, \sigma)=m(t, \tau, s, r) \equiv 0, \quad n(t, \tau, s, \sigma, r)=n(s, \sigma)
$$

this boundary value problem was studied in [1].

We first consider the case $Q=(0,1] \times[-1,1]$. Suppose that $A(\tau)$ is locally integrable in the space $\mathcal{L}^{r}(X)$. As we have shown in Theorem 1, the Cauchy operator $U\left(\tau, \tau_{0}\right)$ for the differential equation (3) may always be written in the form

$$
\begin{aligned}
U\left(\tau, \tau_{0}\right) u(t, s)= & e\left(t, \tau, \tau_{0}, s\right) u(t, s) \\
& +\int_{-1}^{1} \hat{l}\left(t, \tau, \tau_{0}, s, \sigma\right) u(t, \sigma) d \sigma \\
& +\int_{-1}^{1} \hat{m}\left(t, \tau, \tau_{0}, s, r\right) u(r, s) d r \\
& +\int_{-1}^{1} \int_{-1}^{1} \hat{n}\left(t, \tau, \tau_{0}, s, \sigma, r\right) u(r, \sigma) d \sigma d r
\end{aligned}
$$

where $\hat{l}, \hat{m}$ and $\hat{n}$ are measurable. For $f \in C_{r}(X)$, the (unique) solution of equation (3) with initial condition $u\left(\tau_{0}\right)=u_{\tau_{0}} \in X$ is then given by

$$
u(\tau)=U\left(\tau, \tau_{0}\right) u_{\tau_{0}}+\int_{\tau_{0}}^{\tau} U(\tau, \theta) f(\theta) d \theta
$$

Let

$$
u_{r_{1}}=\left\{\begin{array}{ll}
\varphi & \text { on }(0,1] \times[-1,1] \\
y & \text { on }[-1,0) \times[-1,1]
\end{array} \quad \text { and } \quad u_{r_{2}}= \begin{cases}z & \text { on }(0,1] \times[-1,1] \\
\psi & \text { on }[-1,0) \times[-1,1]\end{cases}\right.
$$


where $y$ and $z$ will be specified later. For $\tau_{0}=\tau_{1}$ we get then

$$
\begin{aligned}
& u(t, \tau, s)= \\
& \left\{\begin{array}{l}
m_{1}(t, \tau, s)+\int_{-1}^{0} \hat{m}\left(t, \tau, \tau_{1}, s, r\right) y(r, s) d r \\
+\int_{-1}^{0} \int_{-1}^{1} \hat{n}\left(t, \tau, \tau_{1}, s, \sigma, r\right) y(r, \sigma) d \sigma d r \\
e\left(t, \tau, \tau_{1}, s\right) y(t, s)+\int_{-1}^{1} \hat{l}\left(t, \tau, \tau_{1}, s, \sigma\right) y(t, \sigma) d \sigma \\
+m_{2}(t, \tau, s)+\int_{-1}^{0} \hat{m}\left(t, \tau, \tau_{1}, s, r\right) y(r, s) d r \\
+\int_{-1}^{0} \int_{-1}^{1} \hat{n}\left(t, \tau, \tau_{1}, s, \sigma, r\right) y(r, \sigma) d \sigma d r
\end{array} \quad \text { if }(t, s) \in(0,1] \times[-1,1]\right. \\
&
\end{aligned}
$$

where

$$
\begin{aligned}
m_{1}(t, \tau, s)= & e\left(t, \tau, \tau_{1}, s\right) \varphi(t, s) \\
& +\int_{-1}^{1} \hat{l}\left(t, \tau, \tau_{1}, s, \sigma\right) \varphi(t, \sigma) d \sigma+\int_{0}^{1} \hat{m}\left(t, \tau, \tau_{1}, s, r\right) \varphi(r, s) d r \\
& +\int_{0}^{1} \int_{-1}^{1} \hat{n}\left(t, \tau, \tau_{1}, s, \sigma, r\right) \varphi(r, \sigma) d \sigma d r+\int_{\tau_{1}}^{r} U(\tau, \theta) f(\theta)(t, s) d \theta \\
m_{2}(t, \tau, s)= & \int_{0}^{1} \hat{m}\left(t, \tau, \tau_{1}, s, r\right) \varphi(r, s) d \\
& +\int_{0}^{1} \int_{-1}^{1} \hat{n}\left(t, \tau, \tau_{1}, s, \sigma, r\right) \varphi(r, \sigma) d \sigma d r+\int_{r_{1}}^{r} U(\tau, \theta) f(\theta)(t, s) d \theta .
\end{aligned}
$$

Using now also the boundary condition (15) for $\tau=\tau_{2}$, we arrive at the following system:

$$
\begin{aligned}
z(t, s)= & m_{1}\left(\dot{t}, \tau_{2}, s\right) \\
& +\int_{-1}^{0} \hat{m}\left(t, \tau_{2}, \tau_{1}, s, r\right) y(r, s) d r+\int_{-1}^{0} \int_{-1}^{1} \hat{n}\left(t, \tau_{2}, \tau_{1}, s, \sigma, r\right) y(r, \sigma) d \sigma d r
\end{aligned}
$$


if $(t, s) \in(0,1] \times[-1,1]$ and

$$
\begin{aligned}
\psi(t, s)= & e\left(t, \tau_{2}, \tau_{1}, s\right) y(t, s)+m_{2}\left(t, \tau_{2}, s\right) \\
& +\int_{-1}^{1} \hat{l}\left(t, \tau_{2}, \tau_{1}, s, \sigma\right) y(t, \sigma) d \sigma+\int_{-1}^{0} \hat{m}\left(t, \tau_{2}, \tau_{1}, s, r\right) y(r, s) d r \\
& +\int_{-1}^{0} \int_{-1}^{1} \hat{n}\left(t, \tau_{2}, \tau_{1}, s, \sigma, r\right) y(r, \sigma) d \sigma d r
\end{aligned}
$$

if $(t, s) \in[-1,0) \times[-1,1]$. To simplify this we put, for $(t, s) \in[-1,0] \times[-1,1]$,

$$
\begin{aligned}
& \hat{l}(t, s, \sigma)=-\frac{\hat{l}\left(t, \tau_{2}, \tau_{1}, s, \sigma\right)}{e\left(t, \tau_{2}, \tau_{1}, s\right)} \\
& \tilde{n}(t, s, \sigma, r)=-\frac{\hat{n}\left(t, \tau_{2}, \tau_{1}, s, \sigma, r\right)}{e\left(t, \tau_{2}, \tau_{1}, s\right)} . \\
& \tilde{m}(t, s, r)=-\frac{\hat{m}\left(t, \tau_{2}, \tau_{1}, s, r\right)}{e\left(t, \tau_{2}, \tau_{1}, s\right)} \\
& \tilde{f}(t, s)=\frac{\psi(t, s)-m_{2}\left(t, \tau_{2}, s\right)}{e\left(t, \tau_{2}, \tau_{1}, s\right)} .
\end{aligned}
$$

The above system may then in turn be written as a single equation

$$
\begin{aligned}
y(t, s)= & \int_{-1}^{1} \tilde{l}\left(t, \tau_{2}, \tau_{1}, s, \sigma\right) y(t, \sigma) d \sigma+\int_{-1}^{0} \tilde{m}\left(t, \tau_{2}, \tau_{1}, s, r\right) y(r, s) d r \\
& +\int_{-1}^{0} \int_{-1}^{1} \tilde{n}\left(t, \tau_{2}, \tau_{1}, s, \sigma, r\right) y(r, \sigma) d \sigma+\tilde{f}(t, s)
\end{aligned}
$$

for $(t, s) \in[-1,0] \times[-1,1]$. This is a partial integral equation of the second kind. If $A$ is of Volterra type this equation is also of Volterra type, by Theorem 1 above, and has been studied in [6]. It turns out that in this case the equation (17) is usually uniquely solvable.

Now we consider a general measurable set $Q \subseteq[-1,1] \times[-1,1]$. We denote by $X_{Q}$ the subspace of $X$ consisting of functions with support in $Q$, and by $X_{Q^{c}}$ its complemented subspace.

The proof of the following lemma follows from a straightforward calculation. Recall that an operator function $A=A(\tau)$ is strongly continuous if $\tau \mapsto A(\tau) x$ is continuous for any $x$.

Lemma 3. Let $l=m=n=0, \varphi \in X_{Q}$, and $\psi \in X_{Q^{c}}$. Suppose that the operator function (5) is strongly continuous and measurable in $\mathcal{L}(X)$. Then the boundary value problem (1), (14), (15) has for each $f \in C_{\tau}(X)$ a unique solution in $C_{\tau}^{1}(X)$. This solution is given by

$$
g(t, \tau, s)= \begin{cases}e\left(t, \tau, \tau_{1}, s\right) \varphi(t, s)+P f(t, \tau, s) & \text { if }(t, s) \in Q \\ e\left(t, \tau, \tau_{2}, s\right) \psi(t, s)+P f(t, \tau, s) & \text { if }(t, s) \in Q^{c}\end{cases}
$$


where we have put

$$
P f(t, \tau, s)= \begin{cases}\int_{\tau_{1}}^{\tau} e(t, \tau, \theta, s) f(t, \theta, s) d \theta & \text { if }(t, s) \in Q \\ \int_{\tau_{2}}^{\tau} e(t, \tau, \theta, s) f(t, \theta, s) d \theta & \text { if }(t, s) \in Q^{c}\end{cases}
$$

Lemma 4. Let $K(\tau)=L(\tau)+M(\tau)+N(\tau)$ be defined as in $(6)-(8)$ and $\hat{K}$ on $C_{r}(X)$ be defined by $\hat{K} u(t, \tau, s)=K(\tau) u(\tau)(t, s)$. Suppose that the operator function $K(\tau)$ is strongly continuous in $\mathcal{L}(X)$. Then $\hat{K}$ maps the space $C_{\tau}(X)$ into itself.

Proof. For any $u \in C_{\mathrm{r}}(X)$ we have

$$
\left\|\hat{K} u(\cdot, \tau, \cdot)-\hat{K} u\left(\cdot, \tau_{0}, \cdot\right)\right\| \leq\left\|K(\tau)\left(u(\tau)-u\left(\tau_{0}\right)\right)\right\|+\left\|\left(K(\tau)-K\left(\tau_{0}\right)\right) u\left(\tau_{0}\right)\right\| .
$$

The right-hand side of this inequality tends to zero, as $\tau \rightarrow \tau_{0}$, by the uniform boundedness principle and the strong continuity of the operator function $K(\tau)$

Theorem 3. Assume that the operator functions $C(\tau)$ and $K(\tau)$ are strongly continuous and measurable in $\mathcal{L}(X)$, and let $f \in C_{r}(X)$. Then each solution of the boundary value problem (1),(14),(15) is a solution of the fixed point equation

$$
u(t, \tau, s)=P \hat{K} u(t, r, s)+g(t, \tau, s) .
$$

Conversely, every solution $u \in C_{\tau}(X)$ of equation (18) actually belongs to $C_{\tau}^{1}(X)$ and solves the boundary value problem (1),(14),(15).

Proof. Suppose that $u \in C_{\boldsymbol{\tau}}(X)$ solves problem (1), (14), (15). Replace $f$ in Lemma 3 by $\tilde{f}=\hat{K} u+f$. Since, by Lemma $4, \tilde{f} \in C_{\tau}(X)$, the $C_{r}^{1}(X)$-solution of equation

$$
\frac{\partial u}{\partial \tau}(t, \tau, s)=c(t, \tau, s) u(t, \tau, s)+\tilde{f}(t, \tau, s)
$$

with boundary conditions (14) and (15) is given by

$$
\tilde{g}(t, \tau, s)=P \hat{K} u(t, \tau, s)+g(t, \tau, s)=u(t, \tau, s) .
$$

For the conversion, let $u \in C_{r}(X)$ solve equation (19). Since again $\tilde{f} \in C_{\tau}(X)$, by Lemma 3, the initial value problem has the $C_{r}^{1}(X)$-solution (19)

Lemma 5. Under the assumptions of Theorem 3, the operators $P$ and $P \hat{K}$ map $C_{r}(X)$ into $C_{\tau}^{1}(X)$. Furthermore,

$$
\begin{gathered}
\frac{d P u}{d \tau}(\cdot, \tau, \cdot)=u(\cdot, \tau, \cdot)+C(\tau) P u(\cdot, \tau, \cdot) \\
\frac{d P \hat{K} u}{d \tau}(\cdot, \tau, \cdot)=\hat{K} u(\cdot, \tau, \cdot)+C(\tau) P \hat{K} u(\cdot, \tau, \cdot) .
\end{gathered}
$$

Proof. By Lemma 4 it suffices to consider $P$. Let $f=u \in C_{\tau}(X)$ and $\varphi=\psi=0$. By Lemma 3 there exists a solution $g \in C_{\tau}^{1}(X)$ of the equation

$$
\frac{\partial g}{\partial \tau}(t, \tau, s)=c(t, \tau, s) g(t, \tau, s)+u(t, \tau, s)
$$

namely $g=P u$. This shows that $P u \in C_{r}^{1}(X)$ as claimed 
From Lemma 5 we conclude that the operator $B=P \hat{K}$ maps $C(J, X)$ into itself. Thus we may estimate the spectral radius of $B$ :

Theorem 4. Suppose that the conditions of Theorem 3 are satisfied, and $K=K(\tau)$ is locally integrable in $\mathcal{L}^{r}(X)$. Assume, in addition, that for almost all $(t, \tau, s)$ we have

$$
c(t, \tau, s) \leq 0 \quad((t, s) \in Q) \quad \text { and } \quad c(t, \tau, s) \geq 0 \quad\left((t, s) \in Q^{c}\right)
$$

and

$$
\int_{\tau_{1}}^{r_{2}} \cdots \int_{r_{1}}^{r_{2}}\left\|\left|K\left(\theta_{1}\right)\right| \cdots\left|K\left(\theta_{n}\right)\right|\right\| d \theta_{n} \cdots d \theta_{1}<1
$$

for some $n$. Then the boundary value problem (1),(14),(15) has a unique solution $u \in C_{\tau}^{1}(X)$ for any $\varphi \in X_{Q}, \psi \in X_{Q^{c}}$, and $f \in C_{\tau}(X)$.

Proof. It suffices to prove that the spectral radius $r(B)$ of the operator $B=P \hat{K}$ in the space $C(J, X)=C_{\tau}(X)$ is strictly less than 1 . For any $u \in C_{\tau}(X)$ we have

$$
|B u(t, r, s)| \leq \int_{\tau_{1}}^{\tau_{2}}|K(\theta)||u(\cdot, \theta, \cdot)|(t, s) d \theta .
$$

By induction, we get

$$
\left|B^{n} u(t, \tau, s)\right| \leq \int_{r_{1}}^{r_{2}} \cdots \int_{r_{1}}^{r_{2}}\left|K\left(\theta_{1}\right)\right| \cdots\left|K\left(\theta_{n}\right)\right|\left|u\left(\cdot, \theta_{n}, \cdot\right)\right|(t, s) d \theta_{n} \cdots d \theta_{1} .
$$

This implies that, for almost all $\tau$,

$$
\begin{aligned}
\left\|B^{n} u(\cdot, \tau, \cdot)\right\| & \leq\left\|\int_{r_{1}}^{r_{2}} \cdots \int_{r_{1}}^{r_{2}}\left|K\left(\theta_{1}\right)\right| \cdots\left|K\left(\theta_{n}\right)\right|\left|u\left(\cdot, \theta_{n}, \cdot\right)\right| d \theta_{n} \cdots d \theta_{1}\right\| \\
& \leq\left(\int_{r_{1}}^{r_{2}} \cdots \int_{r_{1}}^{r_{2}}\left\|\left|K\left(\theta_{1}\right)\right| \cdots\left|K\left(\theta_{n}\right)\right|\right\| d \theta_{n} \cdots d \theta_{1}\right)\|u\|_{C\left(J_{1} X\right) .}
\end{aligned}
$$

We conclude that $r(B) \leq\left\|B^{n}\right\|^{1 / n}<1$ in $C(J, X)$

We close with an example which shows that in general the boundary value problem (1), (14), (15) need not have a unique solution.

Example 2. Let $J=\left[0, \frac{\pi}{2}\right]$ and

$$
l(s, \sigma)= \begin{cases}0 & \text { if } s, \sigma>0 \text { or } s, \sigma<0 \\ 1 & \text { if } s>0 \text { and } \sigma<0 \\ -1 & \text { if } s<0 \text { and } \sigma>0\end{cases}
$$


Consider the boundary value problem

$$
\begin{aligned}
\frac{\partial u(t, \tau, s)}{\partial \tau} & =\int_{-1}^{1} l(s, \sigma) u(t, \tau, \sigma) d \sigma & \left((t, \tau, s) \in[-1,1] \times\left[0, \frac{\tau}{2}\right] \times[-1,1]\right) \\
u(t, 0, s) & =0 \quad(s>0) \quad \text { and } \quad & u\left(t, \frac{\pi}{2}, s\right)=0 \quad(s<0) .
\end{aligned}
$$

Apart from the trivial solution $u(t, \tau, s) \equiv 0$, a non-trivial solution is then given by

$$
u(t, \tau, s)= \begin{cases}\sin \tau & \text { if } s>0 \\ \cos \tau & \text { if } s<0\end{cases}
$$

\section{References}

[1] Appell, J., Kalitvin, A. S., and P. P. Zabrejko: Boundary value problems for integrodifferential equations of Barbashin type. J. Int. Equ. Appl. 6 (1994), 1 - 30.

[2] Burgess, D. C. J.: Abstract moment problems with applications to the $l^{p}$ and $L^{p}$ spaces. Proc. London Math. Soc. 4 (1954), 107 - 128.

[3] Case, K. M. and P. F. Zweifel: Linear Transport Theory. Reading, Mass.: Addison-Wesley 1967.

[4] Daletskii, Ju. L. and M. G. Krein: Stability of Solutions of Differential Equations in Banach Space (Transl. Math. Monographs: Vol. 43). Providence, R.I.: Amer. Math Soc. 1974.

[5] Hille, E. and R. S. Phillips: Functional Analysis and Semi-Groups (Coll. Publ.). Providence, R.I.: Amer. Math. Soc. 1957.

[6] Kalitvin, A. S. and P. P. Zabrejko: On the theory of partial integral operators. J. Int. Equ. Appl. 3 (1991), 351 - 382.

[7] Minin, I. N.: Theory of Radiation Transfer in the Atmosphere of Planets (in Russian). Moscow: Nauka 1988.

[8] Rao, M. M. and Z. D. Ren: Theory of Orlicz Spaces. New York: M. Dekker 1991.

[9] Sobolev, V. V.: The Transfer of Radiation Energy in the Atmosphere of Stars and Planets (in Russian). Moscow: Gostekhizdat 1956.

[10] Zabrejko, P. P.: Nonlinear integral operators (in Russian). Voronezh. Gos. Univ. Trudy Sem. Funk. Anal. 8 (1966), 1 - 148.

[11] Zabrejko, P. P.: Ideal function spaces (in Russian). Part I. Jarosl. Gos. Univ. Vestnik 8 (1974), $12-52$.

Received 14.02.1995 